\title{
AN FPGA IMPLEMENTATION OF A DENOISIG ALGORITHM BASED ON STATIONARY WAVELET PACKET TRANSFORN
}

\author{
Nivin A. Ghamry, PhD \\ Electrical and Electronics Department, Faculty of Engineering, \\ Elfayyoum University \\ nivinghamry@yahoo.com
}

(Received June 22, 2006 Accepted July 26, 2006)

\begin{abstract}
Scientific datasets are often contaminated with noise, either because of the data acquisition and transmission processes, or because of naturally occurring phenomena such as atmospheric disturbances. A first pre-processing step in analyzing such datasets is denoising, that is, estimating the signal of interest from the available noisy data. Wavelet transforms represent signals with a high degree of sparsity. This is the principle behind the non-linear wavelet based signal denoising technique and is what distinguishes it from entire linear denoising techniques such as least squares. It is well known that the use of non-decimated (Stationary) Wavelet Transforms (SWT) gives a redundant representation of an input signal, which minimizes the artifacts in the reconstructed data. In this work the stationary wavelet basis and the Wavelet Packet Transform (WPT) method are exploited to develop a Stationary Wavelet Packet Transform (SWPT)-based denoising algorithm. The decomposition of noisy signals is performed with stationary wavelets according to the optimum decomposition tree structure, determined through the WPT method. The thresholding is performed on the coefficients of the best tree, to make the denoising process more efficient. The performance of the denoising algorithm is assessed in terms of the Mean Squared Error (MSE) as a measure of the quality of denoisign. The obtained simulation results indicate that the combination of the SWT and WPT achieves superior denoising than the application of each of them separately. The proposed SWPT-based denoising algorithm is efficiently implemented on Xilinx Virtex Field Programmable Gate Array (FPGA).
\end{abstract}

INDEX TERMS: Denoising, stationary wavelets, wavelet packet transform, FPGA.

\section{INTRODUCTION}

Wavelet denoising has been recently introduced and has found efficient applications in restoration of different types of signals, ranging from medical imaging, astronomy and computer vision to synthetic aperture radar. The orthogonal discrete wavelet transform DWT has high energy compaction and decor relation properties, which make most of the energy of the processed original signal be compacted into a few high magnitude 
coefficients. If the input signal is corrupted by additive white noise, components which correspond to noise will be distributed among low magnitude high frequency components. Hence, a shareholding algorithm in the orthogonal transform domain will remove most of the noise components, while features in the original signal remain sharp after denoising. Various examples show how the noise is largely suppressed by the above approach in contrast with traditional linear methods of smoothing which trade-off noise suppression against a broadening of signal features. Authors addressed the problem of denoising and retrieval of signals buried in excessive noise extensively. The early work of Donohue and Johnston [1] and [2] as well as the work of Chang [3] exploited the decomposition of the data into the orthogonal wavelet basis and gave rigorous justification of denoising via coefficient shareholding. Other authors used stationary wavelets transform for denoising such as the work in [4], [5] and recently in [6], in which they showed the capability of SWT to offer better denoising performance than the ordinary orthogonal wavelets. Other methods were proposed using different wavelet-based operators, such as the wavelet packet transform. Improved results in the area of speech and image coding, as well as signal detection and identification, have been reported using this kind of wavelet analysis [7]. In [8] a WPT-based denoising technique was introduced to serve as a preprocessor for compensation for hearing impairments in hearing aids design. In [9] the author presented the FPGA implementation of the algorithm. The implementation was based on folded-serial word architecture with a high degree of complicity. SWPT method for denoising is yet less exploited than the previous methods as a literature survey on the existing methods shows. FPGA implementations of an algorithm for denoising based on orthogonal SWPT are given in [10] and [11]. For the aim of hardware area reduction in that implementations, the filter coefficients were quantized, which affected the selectivity of the filters. The goal of this work is to further address the issue of combining the use of the wavelet packet method and the stationary wavelet analysis for denoising, exploiting the features of each method. The decomposition and best tree selection of a signal corrupted with additive Gaussian white noise are performed in the biorthogonal basis. An adaptive threshoding method based on local thresholding is applied on the wavelet detail coefficients, before the clean signal can be reconstructed. The proposed algorithm is efficiently implemented on Xilinx FPGA. The dyadic biorthogonal filter coefficients are realized with shift registers without quantization to preserve selectivity. The mentor Graphics tools are applied for design capture in VHDL, simulation and synthesis. Device utilization report including area occupation and operating frequency is provided.

The organization of the rest of this paper is as follows. Section 2 is a brief introduction to the concepts and theory of stationary wavelet transform and wavelet packet transforms algorithms. In section 3 the proposed denoising algorithm combining both criteria is presented. Simulations are run on Matlab and MSE is calculated to assess the efficiency of the denoising algorithm objectively and subjectively. In section 4 the proposed algorithm is implemented on FPGA. Finally, the conclusion and summary of the paper are given in section 5 .

\section{THE PRINCIPLES OF SWT AND WPT}

In this section a brief overview on the principles and theory of the SWT and WPT is given to emphasize the features of each method. 


\section{A. Stationary Wavelet Transform}

Although the classical Discrete Wavelet Transform (DWT) wavelet transform has been shown to offer effective denoising performance, it suffers the drawback, that it is not a time- invariant transform. This means that a translation of the original signal does not necessarily imply a translation of the corresponding wavelet coefficients. The translation invariance property is useful for several important applications, such as breakdown point estimation and detection. To restore this desirable property lost by the classical DWT some slightly different DWT, called the Stationary Wavelet Transform (SWT) is introduced [12]. The SWT algorithm is close to the DWT one, but the downsampling operation is suppressed. The decomposition obtained is then a redundant representation of the signal. The benefit of this redundant representation over the memory-efficient decimated DWT is the reduction of artifacts at discontinuities and irregularities in reconstructed signals. These artifacts are caused by unpredictable changes in coefficients with different time shifts. In practice, the structure of the cascaded filter bank does not change. More precisely, for the first level of decomposition, the SWT coefficients for a given signal are obtained by convolving the input signal with the appropriate filters as in the DWT case but without downsampling. The approximation and detail coefficients at the first level are of the same length as the input signal The general decomposition step $j+1$ convolves the approximation coefficients at level $\mathrm{j}$, with upsampled versions of the appropriate original filters, to produce the approximation and detail coefficients at level $j+1$. The filters are dilated by inserting zeros between the filter coefficients of the previous scale. This can be visualized by Figure 1. Figure 1(a) shows the general decomposition step and Figure 1(b) shows the stationary wavelet filter calculations. $\mathrm{CA}_{\mathrm{j}}$ and $\mathrm{CD}_{\mathrm{j}}$ represent the approximation and detail coefficients of level $j$, respectively and $\mathrm{H}_{j}$ and $\mathrm{G}_{\mathrm{j}}$ represent the low- and high pass decomposition filters at the same level.

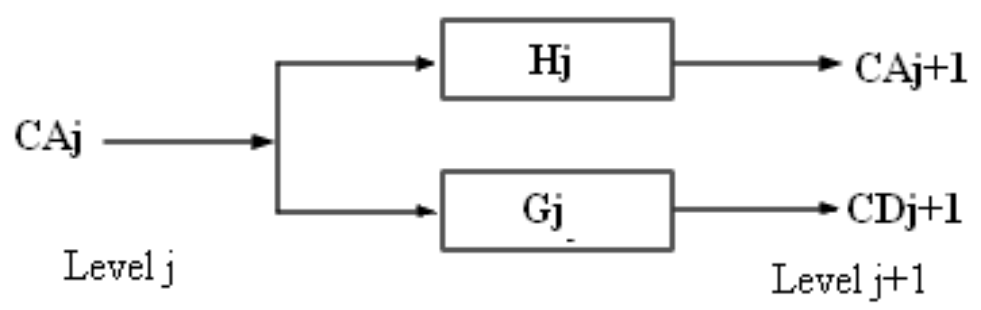

(a)
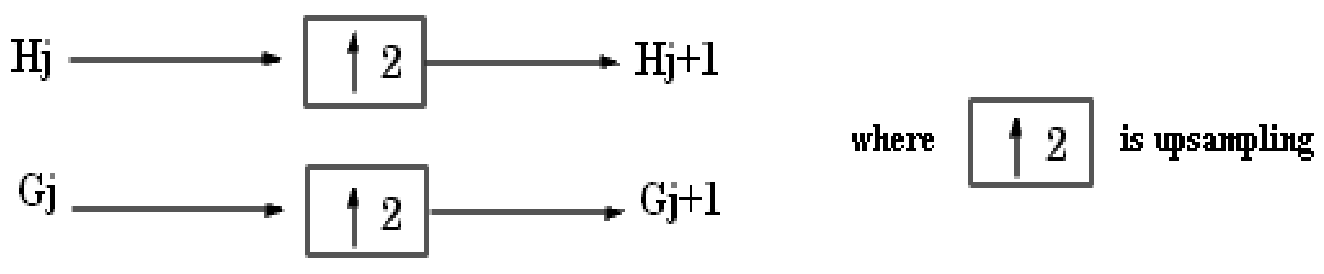

Figure 1: (a) Stationary Wavelet Decomposition.

(b) Stationary Wavelet Filter Calculation. 
As the stationary wavelet coefficients are generated by many different bases, the Inverse Stationary Wavelet Transform (ISWT) operator is not unique and different methods can be used to perform reconstruction. Certainly, the inverse transform results are same. The idea of the inverse discrete stationary wavelet transform is to average the inverses obtained for every non-decimated DWT. This can be done recursively, starting from level $\mathrm{j}$ down to level 1 . In this way the main features of the analyzed signal is captured. One important application of the SWT is denoising.

\section{B. Wavelet Packet Transform}

The wavelet packet method is a generalization of wavelet decomposition that offers a richer range of possibilities for signal analysis. WPT was first introduced by Coifman et al. [13] and [14] for dealing with the non stationarity of the data in a better manner than DWT does. In wavelet packet analysis, the details as well as the approximations can be split to give the wavelet packet decomposition tree. The ordinary wavelet decomposition tree is a part of this complete binary tree. The main feature of the wavelet packet analysis is that it allows signal representations that are not possible with ordinary wavelet analysis. The idea of this decomposition is to start from a scaleoriented decomposition, and then to analyze the obtained signals on frequency subbands. Figure 2 shows the structure of a typical wavelet packet decomposition tree.

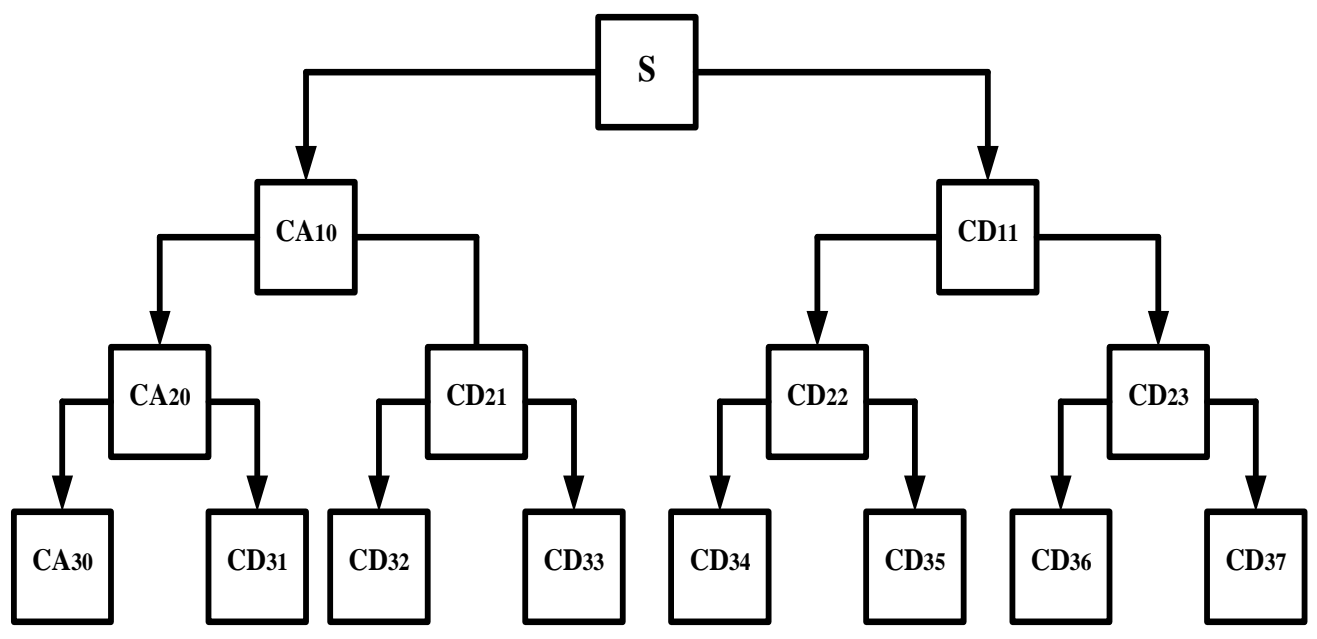

Figure 2: Wavelet Packet Decomposition Tree.

The complete WPT tree is not necessary for perfect reconstruction of the analyzed signal, usually a subtree is chosen based on a data-based cost function. Classical entropy-based criteria are used to select the most suitable decomposition of a given signal, as the number of various signal representations may be very large. This selection is called the best tree structure. Each node of the decomposition tree is examined to quantify the information gained by performing each split. In general a node $\mathrm{N}$ is split into two nodes $\mathrm{N} 1$ and N2 if and only if the sum of the entropy of N1 and $\mathrm{N} 2$ is lower than the entropy of N. This is a local criterion based only on the information available at the node N. Several entropy type criteria can be used to gain 
the optimal decomposition selection. Commonly used entropy types in the field of signal processing are the Shannon entropy, the logarithm of the energy entropy and the threshold entropy. De-noising and compression of signals are interesting applications of wavelet packet analysis.

\section{THE PROPOSED STATIONARY WAVELET PACKET DE-NOISING METHOD}

The general de-noising procedure in the wavelet domain involves three steps, namely:

1. The wavelet decomposition of the noisy signal to a level $n$.

2. The thresholding of the detail coefficients at the different levels with suitable thresholding steps.

3. The reconstruction using the original approximation coefficients and the denoised detail coefficients of levels 1 to $n$.

The thresholding in the second step can be either hard or soft. Hard thresholding can be described as the usual process of setting to zero the elements whose absolute values are lower than the threshold. Soft thresholding is an extension of hard thresholding. It involves first setting to zero the elements whose absolute values are lower than the threshold, and then shrinking the nonzero coefficients towards zero. The proposed denoising method exploits the features of the wavelet packet analysis in the stationary wavelet basis domain for decomposition. To get the optimum representation of the decomposed signal, the best tree selection algorithm is applied on the non-decimated stationary wavelet coefficients. Denoising of the coefficients is achieved via soft thresholding given by the following equation [1]:

$$
T_{j}=\sigma_{j} \sqrt{2 \ln N / 2^{j}}
$$

where $\mathrm{N}$ is the length of the input signal and $\sigma_{j}$ is the standard deviation of noise at scale j. The soft threshold is calculated on a level-dependent basis, rather than global calculation in which one threshold is used for all coefficients (also called fixed form threshold). Here, different thresholds exist for the different multiresolution. The local thresholding criterion increases the capability of the denoising strategies in handling the variance of noise and improves the limits of the classical de-noising strategies. The local thresholding is based on the work of Marc Lavielle about detection of change points using dynamic programming [15]. In this work the B-spline biorthogonal stationary wavelets are chosen for the decomposition of a signal corrupted by additive white noise generated by Maltab wavelet toolbox. The B-spline biorthogonal wavelet families are symmetric, smooth and their coefficients build a bilinear series. This symmetry in FIR filters guarantees linear characteristics for the frequency response and phase, which is in general a very desirable property in signal processing applications.

The following steps summarize the proposed method:

1. The first level of stationary wavelet decomposition is performed to obtain non decimated-wavelet coefficients in the biorthogonal wavelet basis.

2. Each node is checked for further splitting based on the chosen entropy criterion. 
3. In the second (as well as in further) level(s) of decomposition the approximations and/or details are split by convolving them with the upsampled versions of the biorthogonal wavelet filters applied in the first step.

4. Successive splitting of the coefficients and upsampling of the decomposition filters is repeated until the best tree structure at a predefined decomposition level is obtained.

5. For each wavelet packet (except for the approximation coefficients) level dependent thresholding is determined.

6. Soft thresholding is performed on the wavelet coefficients to kill the effect of the noise.

7. Approximations and details are reconstructed from the denoised coefficients.

8. The clean signal is reconstructed from the different approximations and details according to the ISWT algorithm.

The idea of the inverse discrete stationary wavelet transform is to average recursively the inverses obtained form every non-decimated DWT. In this way nothing of the detail information is lost. For the WPT analysis, the Shannon entropy is used for efficient searching to compute the optimal tree after decomposition. Equation (2) for the Shannon entropy E involves the logarithm of the squared value of each signal sample [14]:

$$
E=-\sum_{i} s_{i}^{2} \log \left(s_{i}^{2}\right)
$$

According to the characteristics of wavelet decomposition, the more the decomposition scale is, the better the filtering result, therefore successive splitting of the coefficients and upsampling of the filters is repeated to the fifth level. The selected best tree is shown in Figure 3.

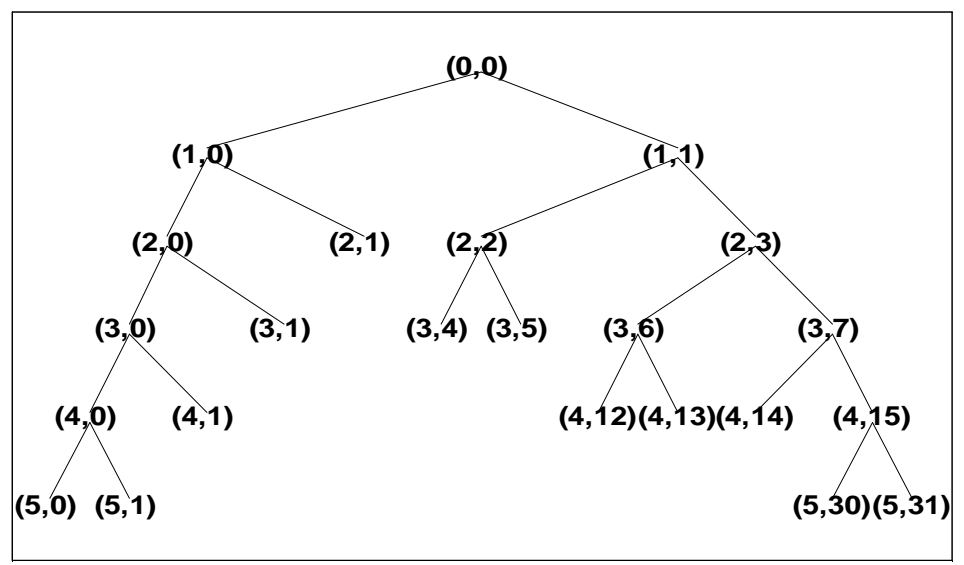

Figure 3: The Selected Best Tree Structure.

For the aim of comparison the denoising of the signal is performed applying SWT and WPT separately prior to applying the SWPT denoising method. The simulation results and calculated MSE are compared. Figure 4(a) shows the original noisy signal $S$ while Figures $\mathbf{4}(\mathbf{b}-\mathbf{d})$ shows the denoised signals applying the various methods. They are 
labeled SWT-S, WPT-S and SWPT-S. Although the result of SWT denoising in Figures $\mathbf{4}(\mathbf{b})$ and show the capability of the SWT to suppress noise from the signal better than the WPT in Figure 4(c) does, the denoised signal is over smoothed, especially in the regions of sharp edges and irregularities. On the other hand, the effect of decimation is obvious in denoising with WPT, as it causes false detection at some intervals, for example at the interval from 200 to 250 . The denoising of the proposed method in Figure $\mathbf{4}(\mathbf{d})$ is more satisfactory as the abrupt changes are detected and preserved and no false detections occur. Furthermore, the residuals look like a white noise sample. The residuals are illustrated in Figure 5.

The calculated MSE are given in Table 1. The values of the MSE support the superiority of the proposed method in denoising over both traditional methods, WPT and SWT as it provides the smallest error.

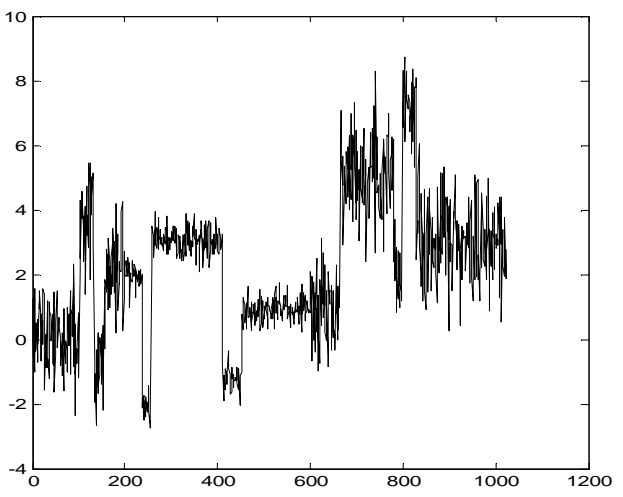

(a)

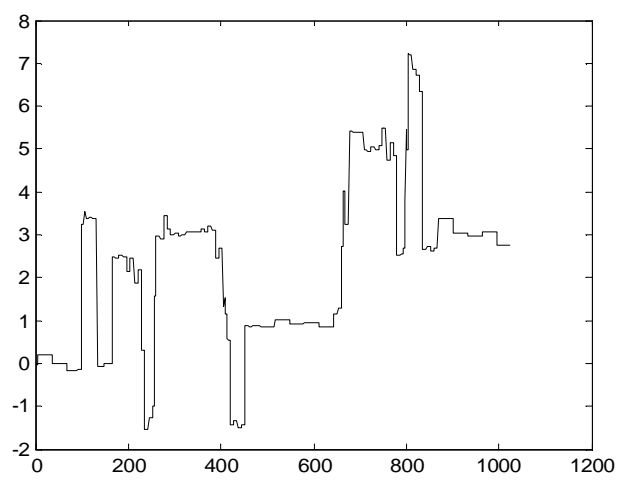

(c)

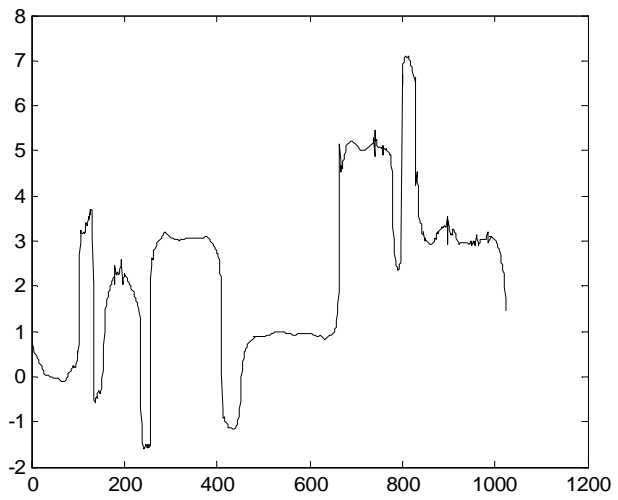

(b)

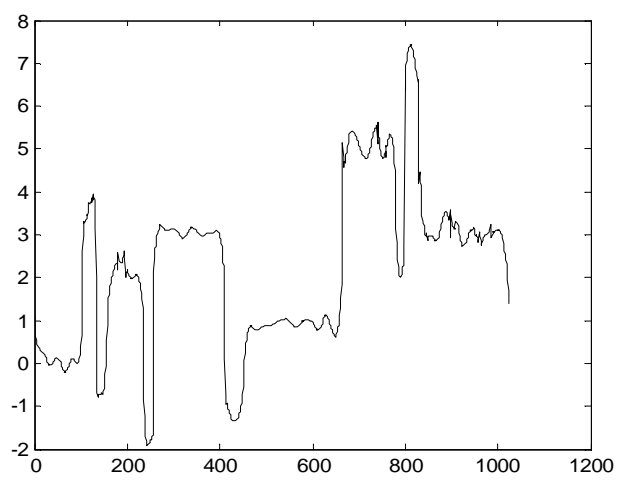

(d)

Figure 4: (a) The Original Noisy Signal S, (b) Denoised Signal with SWT, (c) Denoised Signal with WPT and (d) Denoised Signal with SWPT. 


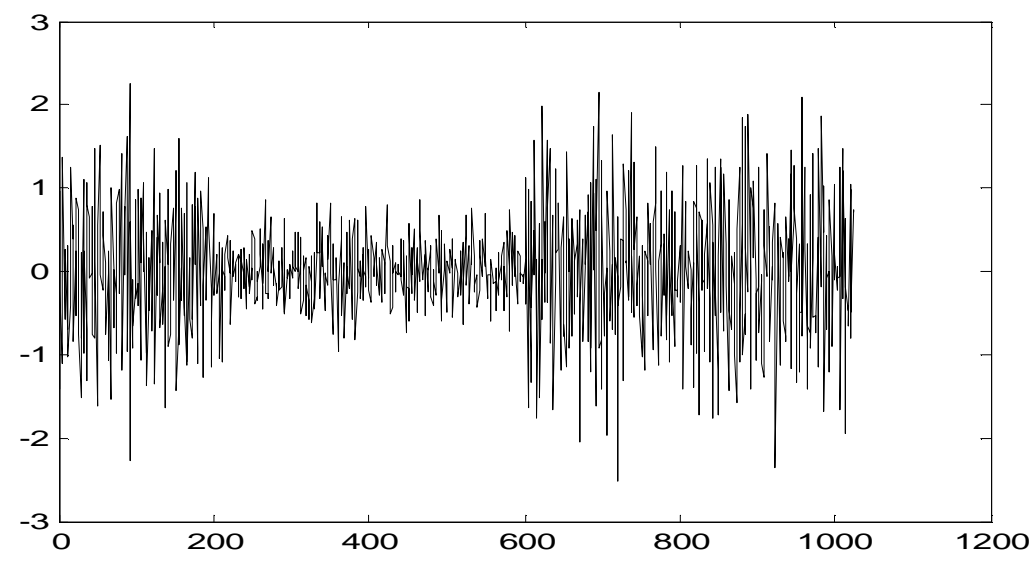

Figure 5: The Residuals.

Table 1: The Calculated MSE.

\begin{tabular}{|c|c|}
\hline Denoising Method & MSE \\
\hline SWT & 25.79 \\
\hline WPT & 31.833 \\
\hline SWPT & 24.69 \\
\hline
\end{tabular}

\section{HARDWARE IMPLEMENTATION}

As mentioned before, the main drawback of the SWT is its heavy computation burden. In the proposed hardware design of the SWPT denoising algorithm, the reduction of the computation burden is achieved as following: Firstly, according to the best tree selection algorithm not all the nodes are split, therefore only the biorthogonal filters corresponding to the best adapted wavelet packet basis are implemented. Multiplexers select the wavelet packet domain to analyze. Secondly, the upsampling of the decomposition filters in further levels is achieved by increasing the number of delay elements in the delay line. Figure 6, shows the hardware implementation schematically. The structure follows the regular filter bank structure suppressing decimation blocks between the successive stages. Storage units and multiplexers are the decoupling elements between the filter modules. An entropy based control unit based on the entropy criterion mentioned before determines the proper select signal for the multiplexers. Accordingly, the coefficients are either split for further decomposition or passed as final outputs. The design is implemented of Xilinx Virtex FPGA. In the following sections the area efficient realization of the decomposition stages is described.

\section{A. First Level Of Decomposition}

For the implementation of the symmetric low- and high pass biorthogonal filters, the multiplierless structure described in [16] is exploited with slight modifications. The symmetric wavelet filter coefficients are given in Table 2. 


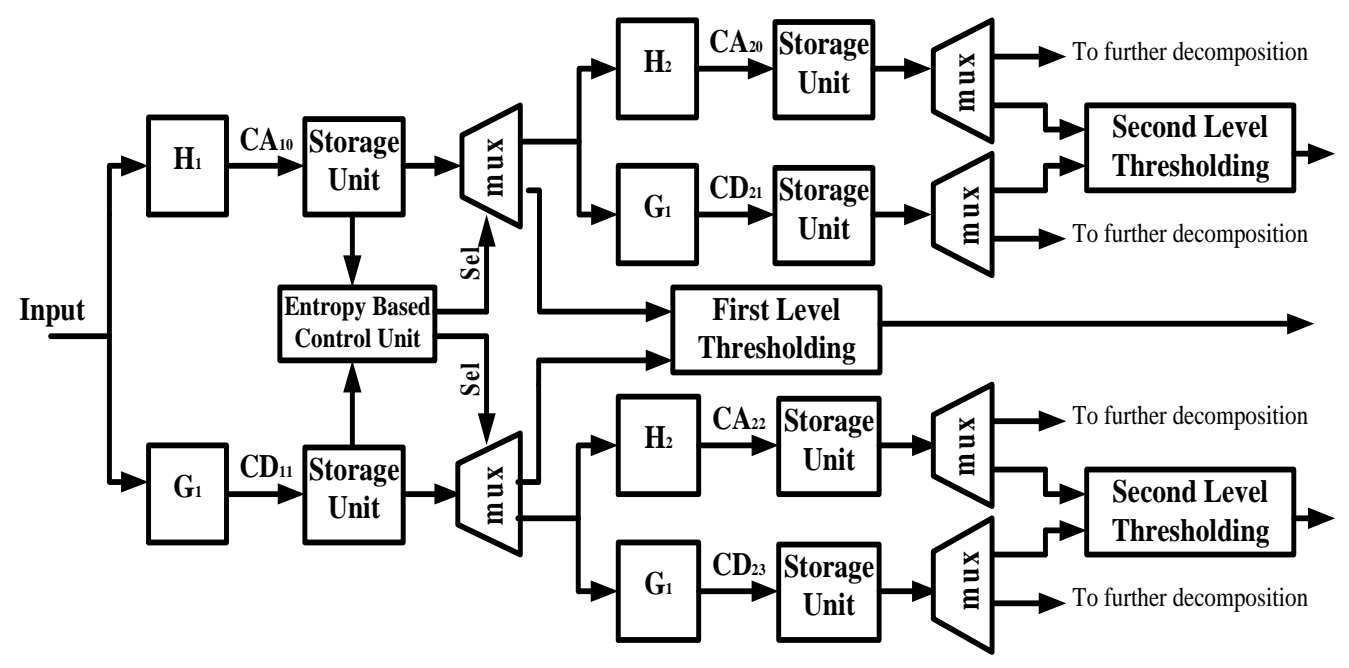

Figure 6: The Block Diagram for the Hardware Implementation.

Table 2: The Wavelet Filter Coefficients .

\begin{tabular}{|c|c|}
\hline Filter Coefficient & Value \\
\hline $\mathrm{H}_{0}$ & -0.0625 \\
\hline $\mathrm{H}_{1}$ & 0.0625 \\
\hline $\mathrm{H}_{2}$ & 0.5000 \\
\hline $\mathrm{H}_{3}$ & 0.5000 \\
\hline $\mathrm{H}_{4}$ & 0.0625 \\
\hline $\mathrm{H}_{5}$ & -0.0625 \\
\hline
\end{tabular}

Figure 7 shows the block diagram of the filter module used for computing the approximation and detail coefficients in one cycle. It consists of a clock generator, a series of delay elements, parallel shift registers, adders and finally an accumulator. The main feature of that design is that it avoids the use of multiplies completely by the use of shift registers instead. The delay elements are used for aligning and synchronizing the input data sequence to the following shift registers. The adders shown are used to sum the input words which are supposed to be multiplied to the same filter coefficients, to decrease the number of shifts. This is a direct benefit of the symmetry of the biorthogonal wavelet filters. The accumulator composes the terminating element of the low pass filters in the design. The outputs of the accumulator are the approximation coefficients. As the high pass filter has only two coefficients it was possible to compute the detail coefficients in the same cycle using only on delay element and a subtractor. The approximation and detail coefficients are now passed to multiplexers to select the following operation, either to split them further or bypass them as final outputs. The select signal to the multiplexer (labeled 'sel') is determined according to the best tree structure. The approximation and detail coefficients are labeled $\mathrm{CA}_{10}$ and $\mathrm{CD}_{10}$, respectively. 


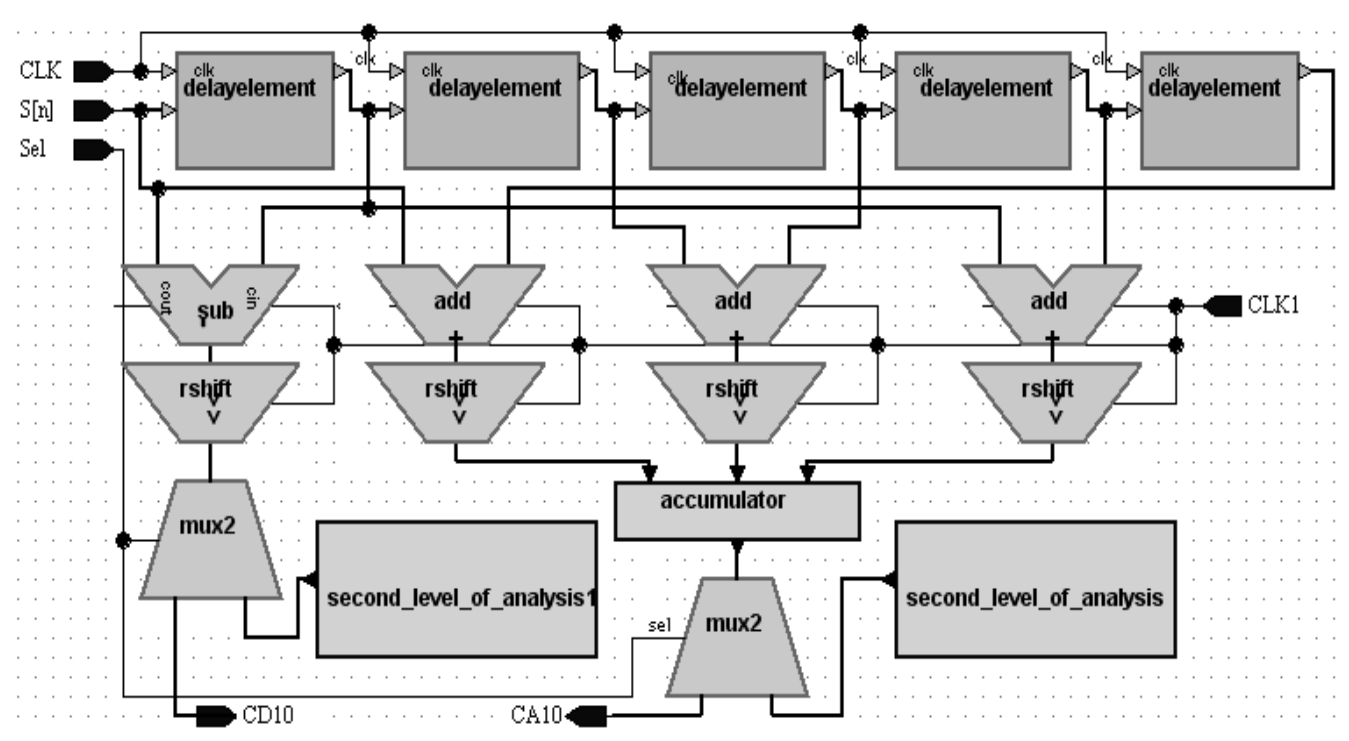

Figure 7: FPGA Implementation of the Filter Modules.

\section{B. Second And Higher Levels Of Decomposition}

For further decomposition of the coefficients the decomposition filters have to be upsampled. To avoid the use of generality in the design of the wavelet filters, the same structure based on adders and shift registers is kept, while the number of delay elements is increased. This can be explained by the equivalence of passing the input data through further delay elements and weighting them with a zero filter coefficients. Accordingly the delay elements of the low- and high pass filters is increased to nine and two, respectively. However the number of adders and shift registers remains as it is.

\section{Features Of FPGA Implementation}

The tools of the Mentor Graphics, FPGA Advantages 5.0 package, are utilized for the simulation and synthesis of the design. The distinct blocks are captured by VHDL, and then, the design structure is represented as graphical block diagrams to ease scalability. After successful compilation, the design is ported to Xilinx Virtex FPGA. The Virtex families are a fast high-volume production FPGA solution that delivers all the key requirements for ASIC replacement. This particular XCV600E chip used has $48 \times 72$ CLB array (row x column) and 985,882 system gates [17]. The storage units for storing the computed coefficients are implemented using the free built-in Block Select RAM incorporated by Virtex E. To get a compact layout and an optimal clock speed, placement and timing constraints are added to the design. The hardware area required for the realization of the complete system for five levels SWPT system is 3022 giving area occupancy of $87 \%$ of the total device. The results are summarized in the device utilization report in Table 3. After synthesis, placement and routing the maximum data throughput is over $30 \mathrm{MHz}$. The obtained results are comparable with the results of the 
work in [10] and [18]. The increase in number of CLB is because more decomposition levels are implemented and because of the on-chip control unit.

Table 3: FPGA Device Utilization Summary

\begin{tabular}{|c|c|c|c|}
\hline Resource & Used & Available & Utilization \% \\
\hline IOBs & 172 & 512 & 36 \\
\hline CLB & 3022 & 3456 & 87 \\
\hline
\end{tabular}

\section{CONCLUSION}

In this work a signal denoising algorithm based on stationary wavelets and wavelet packet transform is developed. The main idea is to select the best tree structure of the decomposed noisy signal in the stationary biorthogonal wavelet domain and apply a local denoising threshold on the detail coefficients. The proposed method as well as the traditional denoising methods SWT and WPT is applied on a noisy test signal. Software simulation results via Matlab proved that the denoising effect of the SWT and WPT is observably enhanced by combining them into the proposed method and is superior to the effect of each method separately. The calculated MSE values supported the simulation results. The algorithm is efficiently implemented on Xilinx Virtex FPGA. In spite of the clearly heavier computation load of stationary wavelet, it was possible to provide VLSI architecture with acceptable area and operating frequency in comparison with other architectures reported in the literature. The main advantages of the proposed architecture is that it is regular, scalable and interconnection follows a simple strategy which facilitates extending it to further decomposition levels by cascading the main building blocks.

\section{REFERENCES}

[1] D.L.Donoho, and I.M. Johnstone, "Ideal spatial adaptation via wavelet shrinkage", Biometrika, Volume 81, pp. 425-455, 1994.

[2] D.L.Donoho, and I.M. Johnstone, "Adapting to unknown smoothness via wavelet shrinkage", Journal of the American Statistical Association, Volume 90, pp. 1200-1224, 1995.

[3] G. Chang, , Yu, B., and M. Vetterli, "Adaptive wavelet thresholding for image denoising and compression”, IEEE Transaction on Image Processing, Volume 9, pp. 1532-1546, 2000.

[4] R.R. Coifman, and D.L.Donoho, "Translation-invariant denoising, in Wavelets and Statistics". Lecture Notes in Statistics, Springer Verlag, pp.125-150, 1995.

[5] L. Qiang, T. Jianfu, W. Xin, Z. Yaqi, G. Ji.Chang, "Research of gyro signal denoising with stationary wavelet", Proceedings of the Third International Conference on Wavelet Analysis and its Applications WAA, May, 2003,

[6] Y. Peng, "Denoising of fast and weak transient signals using selected orthogonal wavelet" Proceedings of the 7th International Conference on Signal Processing, ICSP '04, Volume: 1, pp.260- 263, September, 2004. 
[7] B-L. Lim, Z-L. Ying, "Performance analysis of audio signal compression based on wavelet and wavelet packet transform", Proceedings of the International Conference on Information, Communications and Signal Processing ICICS,pp. 735-739, September,1997.

[8] M. A. Trenas, J. Lbpez, E. L. Zapata, and E Arguello, "An architecture for wavelet-packet based speech enhancement for hearing aids", In Proceedings of the International Conference ICASSP'2000, volume 2, pp. 849-852, June 2000.

[9] M. A. Trenas, J. Lbpez, E. L. Zapata, "FPGA Implementation of Wavelet Packet Transform with Reconfigurable Tree Structure", Proceedings of the 26th Euromicro Conference, Volume: 1, pp.1124-1131, 2000.

[10] M. Montani, L. De Marchi, A. Marcianesi and N. Speciale, "Comparison of a programmable DSP and FPGA implementation of a wavelet-based denoising algorithm, Proceedings of the 46th IEEE International Midwest Symposium on Circuits and Systems, MWSCAS '03, Volume:2, pp.602- 605, 2003.

[11] A. Marcianesi, L. De Marchi, and N. Speciale "Stationary wavelet packet programmable filters for real time signal detection and denoising", Proceeding IASTED Signal Processing, Pattern Recognition, and Applications, 2003.

[12] Nason, G.P.; B.W. Silverman, "The stationary wavelet transform and some statistical applications", Lecture Notes in Statistics, 103, pp. 281-299, 1995.

[13] R.R.; Coifman, M.V Wickerhauser, "Signal processing and compression with wave packets", Numerical Algorithms Research Group, New Haven, CT Yale University, 1990.

[14] R.R.; Coifman, M.V Wickerhauser, "Entropy-based algorithms for best basis selection", IEEE Transaction on Information Theory, Vol. 38, 2, pp. 713-718. 1992.

[15] M. Lavielle, "Detection of multiple changes in a sequence of dependent variables", Stochastic Processes and their Applications, Volume 83, pp. 79-102, Elsevier Science, September, 1999.

[16] Nivin A. Ghamry, "FPGA Implementation of 2-D Biorthogonal Discrete Wavelet Transform", Journal of Ain Shams University, June 2006.

[17] Xilinx virtex-e data sheet, http://www.xilinx.com/partinfo/ds022.pdf

[18] S. Masud, and J. V. McCanny, "Wavelet packet transform for system-on chip applications", Proceedings of the IEEE International Conference on Acoustics, Speech, and Signal Processing ICASSP, Volume 6, pp. 3287 - 3290, 2000 . 


\section{تنفيذ لوغاريتم للتخلص من التشويش باستخدام المويجات الواقفة على

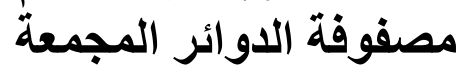

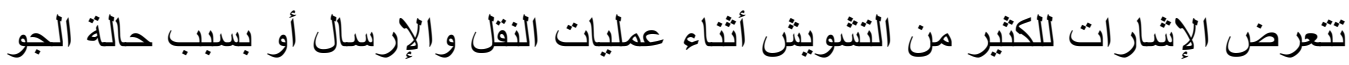

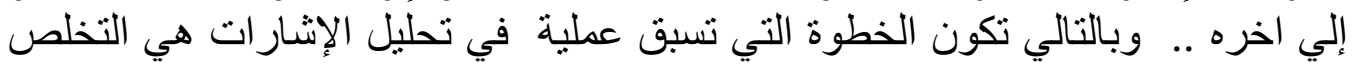

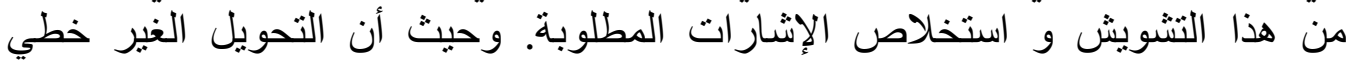

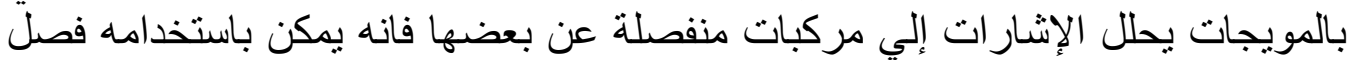

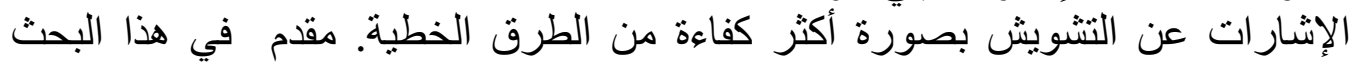

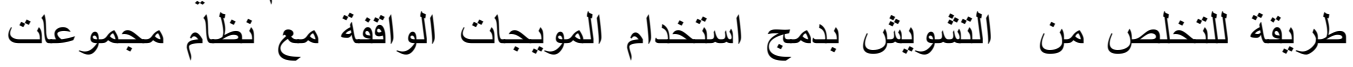

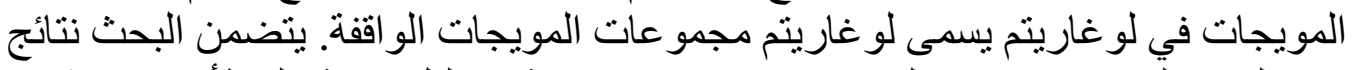

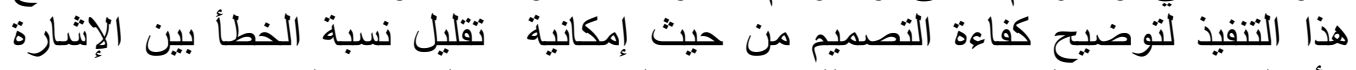

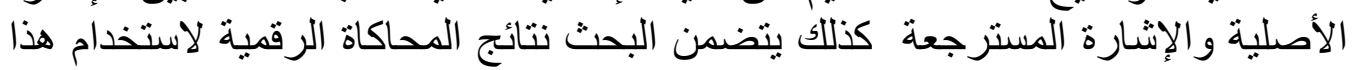

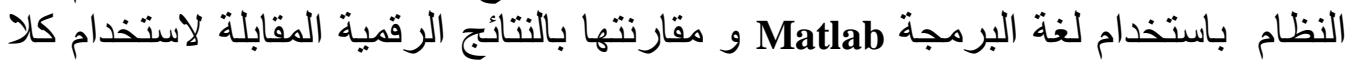

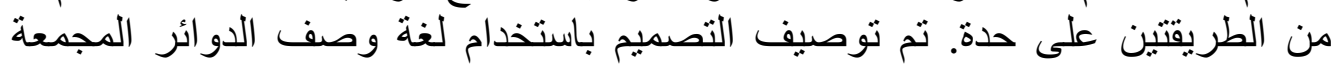

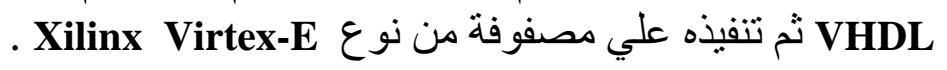

